\title{
Design of near-infrared fluorescent bioactive conjugated functional iron oxide nanoparticles for optical detection of colon cancer
}

This article was published in the following Dove Press journal:

International Journal of Nanomedicine

18 October 2012

Number of times this article has been viewed

\section{Enav Corem-Salkmon \\ Benny Perlstein \\ Shlomo Margel}

The Institute of Nanotechnology and Advanced Materials, Department of Chemistry, Bar-Ilan University, Ramat-Gan, Israel
Correspondence: Shlomo Margel

The Institute of Nanotechnology and Advanced Materials, Department of Chemistry, Bar-llan University, Ramat-Gan, 52900, Israel

Fax +972 36355208

Email shlomo.margel@mail.biu.ac.il
Background: Colon cancer is one of the major causes of death in the Western world. Early detection significantly improves long-term survival for patients with the disease. Near-infrared (NIR) fluorescent nanoparticles hold great promise as contrast agents for tumor detection. NIR offers several advantages for bioimaging compared with fluorescence in the visible spectrum, ie, lower autofluorescence of biological tissues, lower absorbance, and consequently deeper penetration into biomatrices.

Methods and results: NIR fluorescent iron oxide nanoparticles with a narrow size distribution were prepared by nucleation, followed by controlled growth of thin iron oxide films onto cyanine NIR dye conjugated gelatin-iron oxide nuclei. For functionalization, and in order to increase the NIR fluorescence intensity, the NIR fluorescent iron oxide nanoparticles obtained were coated with human serum albumin containing cyanine NIR dye. Leakage of the NIR dye from these nanoparticles into phosphate-buffered saline solution containing $4 \%$ albumin was not detected. The work presented here is a feasibility study to test the suitability of iron oxide-human serum albumin NIR fluorescent nanoparticles for optical detection of colon cancer. It demonstrates that encapsulation of NIR fluorescent dye within these nanoparticles significantly reduces photobleaching of the dye. Tumor-targeting ligands, peanut agglutinin and anticarcinoembryonic antigen antibodies $(\alpha C E A)$, were covalently conjugated with the NIR fluorescent iron oxidehuman serum albumin nanoparticles via a poly(ethylene glycol) spacer. Specific colon tumor detection was demonstrated in chicken embryo and mouse models for both nonconjugated and the peanut agglutinin-conjugated or $\alpha$ CEA-conjugated NIR fluorescent iron oxide-human serum albumin nanoparticles.

Conclusion: Conjugation of peanut agglutinin or $\alpha$ CEA to the nanoparticles significantly increased the fluorescence intensity of the tagged colon tumor tissues relative to the nonconjugated nanoparticles.

Keywords: near-infrared fluorescence, iron oxide nanoparticles, optical imaging, chorioallantoic membrane assay, colon cancer

\section{Introduction}

Over the past 20 years, much has been learned about screening for colon cancer from biological, epidemiological, and clinical studies. Colorectal cancer is a leading cause of death in the Western world, but survival can be improved if the disease is detected at an early stage. ${ }^{1}$ A variety of screening techniques can detect premalignant lesions and early-stage cancers, such as double-contrast barium enema, fecal occult blood tests, and colonoscopy. However, these methods are considered to be either lacking in sensitivity or invasive. . $^{1,2}$ 
Fluorescence imaging, as opposed to white-light imaging, can be used to visualize beyond superficial tissue, and a high signal-to-background ratio can be achieved in the labeled tissue. ${ }^{3}$ A challenge of fluorescence imaging is the significant autofluorescence of bodily tissues in the visible region of the electromagnetic spectrum. Materials with fluorescence in the near-infrared (NIR) region (700-1000 nm) intended for use as imaging agents are of great interest, because they result in a low background signal and relatively deep penetration into biomatrices. ${ }^{4-6}$ However, the wavelengths in the NIR region are beyond those visible to the human eye, and challenges of fluorescence imaging in this region include the need for specialized light sources and detection equipment.

Hsiung et $\mathrm{al}^{7}$ used a synthesized heptapeptide conjugated with fluorescein for detection of human colorectal neoplasia, and tested it in patients undergoing colonoscopy. Imaging of the topically administered peptide was performed with a novel fluorescence confocal microendoscope. Imaging of the fluorescein-conjugated peptide demonstrated preferential binding to dysplastic colonocytes relative to adjacent normal cells. The disadvantage of this procedure is due to the autofluorescence of bodily tissues at the fluorescein wavelength and the photobleaching observed during the imaging procedure when the probe was held stationary on the tissue surface for more than 5 seconds, as reported by Hsiung et al. ${ }^{7}$ Increased interest in developing nanoparticle-based NIR probes has lead to the development of silica, calcium phosphate, and lipoprotein NIR nanoparticles. ${ }^{8-11}$ These nanoparticles have been shown to have significant advantages over organic NIR dyes such as enhanced photostability and an improved fluorescent signal.

Tumors can be targeted actively by nanoparticles conjugated with molecular probes designed to recognize tumor-specific markers. Conjugation of biomolecules to nanoparticles is easily achieved via functional groups on the nanoparticle surface. ${ }^{12-14}$ Known targeting agents include antibodies, lectins, small peptides, and small targeting molecules, all with upregulated receptors on the cell membrane of tumor cells. With targeting agents conjugated to the nanoparticles, the nanoparticles bind to specific receptors and are usually enveloped by endosomes via receptor-mediated endocytosis. Thus, the intracellular concentration of nanoparticles is enhanced in cancer cells compared with normal cells. ${ }^{15}$

The standard assays for evaluating the performance of tumor imaging involve human-tumor xenografts in immunodeficient mice. ${ }^{16}$ Assessment of new imaging agents requires a substantial number of tumor-bearing animals, which is both expensive and time-consuming. We have explored the use of tumors grown on the chorioallantoic membrane (CAM) of embryonic chicken eggs to test the performance of bioactive fluorescent nanoparticles in solid tumors. This system is less expensive, and allows for imaging of tumors in a shorter time period, without use of animals for mandatory in vivo experiments. ${ }^{17}$

Superparamagnetic iron oxide (IO) nanoparticles have a wide range of biomedical applications, such as magnetic resonance imaging contrast agents, drug delivery, specific cell labeling, cell tracking, diagnostics, and hyperthermia. ${ }^{18-22}$ Previously, a unique type of uniform IO nanoparticles was prepared and characterized in our laboratory. ${ }^{23,24}$ These IO nanoparticles were formed by controlled nucleation of IO onto gelatin, dissolved in an aqueous solution, and followed by stepwise growth of thin layers of IO films onto gelatin-IO nuclei. The same IO nanoparticles have been widely investigated in biological applications, and have been shown to be nontoxic. ${ }^{25}$

In the present study, we prepared NIR fluorescent IOhuman serum albumin (HSA) nanoparticles. We substituted the previously used gelatin with gelatin covalently conjugated to a NIR cyanine dye, and the IO nanoparticles obtained were coated with HSA complexed to the cyanine dye. ${ }^{26}$ The NIR fluorescent IO-HSA nanoparticles formed were bioactivated by covalently attaching colon cancer-targeting agents such as peanut agglutinin and anticarcinoembryonic antigen antibodies ( $\alpha$ CEA). ${ }^{27,28}$ These NIR fluorescent bioactive IO nanoparticles were shown to be photostable and demonstrated specific activity in tumor implants in the chicken embryo model and mouse model.

\section{Materials and methods Materials}

The following analytical grade chemicals were purchased from Sigma-Aldrich (Rechovot, Israel) and were used without further purification: 2-[2-[2-chloro-3-[2-[1,3dihydro-3,3-dimethyl-1-(4-sulfobutyl)-2H-indol-2-ylidene] ethylidene]-1 cyclohexen-1-yl] ethenyl]-3,3-dimethyl-1-(4sulfobutyl)-3H-indolium inner salt sodium salt (IR-783), porcine gelatin, glycine, $\mathrm{FeCl}_{2}, \mathrm{NaNO}_{2}, 1 \mathrm{~N} \mathrm{NaOH}, 1 \mathrm{~N}$ $\mathrm{HCl}$, HSA, 4-mercaptobenzoic acid, N,N-dimethylformamide (DMF), 1-ethyl-3-(3-dimethylaminopropyl) carbodiimide (EDC), N-hydroxysulfosuccinimide (sulfoNHS), O,O'-bis[2-(N-succinimidyl-succinylamino)ethyl] polyethylene glycol molecular weight 3000 (NHS-PEGNHS), Matrigel ${ }^{\circledR}$, peanut agglutinin, and fluorescein isothiocyanate-conjugated peanut agglutinin (FITC-PNA). 
Dulbecco`s phosphate-buffered saline (PBS), Eagle's minimum essential medium, fetal bovine serum, glutamine, penicillin, and streptomycin were purchased from Biological Industries (Bet Haemek, Israel). The cell lines LS174T and SW480 were obtained from the American Type Culture Collection (Manassus, VA). Monoclonal anti-CEA antibodies T86-66 ( $\alpha \mathrm{CEA})$ were purified from hybridoma supernatant at Alomone Laboratories, Israel. Water was purified by passing deionized water through an Elgastat Spectrum reverse osmosis system (Elga Ltd, High Wycombe, UK).

\section{Synthesis of CANIR}

Cyanine NIR fluorescent dye (CANIR) was prepared by treatment of IR-783 dye with 4-mercaptobenzoic acid in DMF (see Figure 1), as reported in the literature. ${ }^{29}$ Elemental analysis, mass spectroscopy, and ${ }^{1} \mathrm{H}$ nuclear magnetic resonance showed the $100 \%$ purity of the CANIR dye obtained.

\section{Synthesis of NIR fluorescent IO nanoparticles}

NIR fluorescent iron oxide nanoparticles were prepared by a procedure similar to that described in our previous publication for the preparation of nonfluorescent iron oxide nanoparticles, ${ }^{24}$ substituting the gelatin for CANIR covalently conjugated with gelatin. Briefly, $2 \mathrm{mg}$ of CANIR was dissolved in $1 \mathrm{~mL}$ of water and added to $1 \mathrm{mg}$ of EDC and $2.2 \mathrm{mg}$ of sulfo-NHS dissolved in $1 \mathrm{~mL}$ of water. The mixture was then shaken at room temperature for 15 minutes, and added to $20 \mathrm{~mL}$ of aqueous solution containing $1 \%(\mathrm{w} / \mathrm{v})$ gelatin at $60^{\circ} \mathrm{C} . \mathrm{NaOH}$ $(1 \mathrm{~N})$ was added until a $\mathrm{pH}$ of 8.5 was attained. The mixture was shaken at $60^{\circ} \mathrm{C}$ for one hour. The $\mathrm{pH}$ of the CANIRconjugated gelatin solution was adjusted to 5.0 by adding $\mathrm{HCl}$ $(1 \mathrm{~N})$, followed by further addition of water at $60^{\circ} \mathrm{C}$ to reach a volume of $80 \mathrm{~mL}$. Thereafter, $\mathrm{FeCl}_{2}$ solution $(10 \mathrm{mmol} / 5 \mathrm{~mL}$ $0.01 \mathrm{~N} \mathrm{HCl}$ ) was added to the previous $80 \mathrm{~mL}$ of aqueous solution containing $200 \mathrm{mg}$ of CANIR-conjugated gelatin, followed by $\mathrm{NaNO}_{2}$ solution $(7 \mathrm{mmol} / 5 \mathrm{~mL} \mathrm{H} \mathrm{O}$ ). After a reaction time of 10 minutes, the $\mathrm{pH}$ was raised to 9.5 by adding $\mathrm{NaOH}$ aqueous solution (1N). This procedure was repeated four times. The IO nanoparticles formed were then washed from excess reagents with PBS using a magnetic gradient column. ${ }^{30}$ Non-fluorescent IO nanoparticles were prepared similarly to those of the NIR fluorescent IO nanoparticles, in the absence of the CANIR dye.

\section{Coating of NIR fluorescent IO nanoparticles with HSA}

Coating of the NIR fluorescent IO nanoparticles with HSA (in the absence or presence of CANIR) was accomplished by precipitation of HSA onto the surface of the fluorescent IO nanoparticles. ${ }^{25,31}$ Briefly, $20 \mathrm{mg}$ of HSA and $2 \mathrm{mg}$ of CANIR dissolved in $2 \mathrm{~mL}$ of PBS were added to $10 \mathrm{~mL}$ of fluorescent NIR IO nanoparticles dispersed in PBS $(4 \mathrm{mg} / \mathrm{mL}$, $\mathrm{pH}$ 7.4). The shaken mixture was gradually brought to $65^{\circ} \mathrm{C}$, and after 16 hours at $65^{\circ} \mathrm{C}$ was cooled to room temperature. Excess dye and dissolved albumin were then removed using a magnetic gradient column. An additional coating of pure HSA (in the absence of CANIR dye) was performed similarly by adding $4 \mathrm{mg}$ HSA to $20 \mathrm{mg}$ NIR fluorescent IO-HSA nanoparticles dispersed in PBS (4 mg/mL, pH 7.4). Nonfluorescent IO-HSA nanoparticles were prepared similarly to those of the NIR fluorescent IO-HSA nanoparticles, in the absence of the CANIR dye.

\section{Extent of CANIR leakage}

Leakage of CANIR entrapped in the NIR fluorescent IO nanoparticles into the continuous phase (PBS containing $4 \%$ HSA) was evaluated according to the following procedure: NIR fluorescent IO-HSA nanoparticles dispersed in PBS ( $1 \mathrm{mg} / \mathrm{mL})$ containing 4\% HSA were shaken at room temperature for 4 hours and then filtered via a $300 \mathrm{kDa}$ filtration tube (VS0241 Viva Spin) at $4000 \mathrm{rpm}$

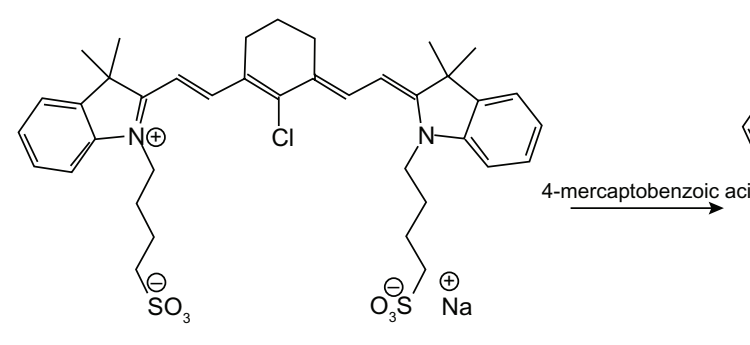

A

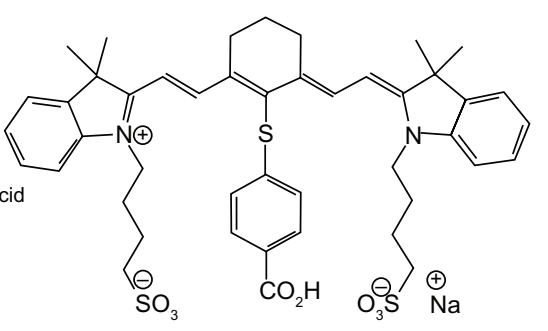

B

Figure I Synthetic scheme describing preparation of cyanine near-infrared fluorescent dye. 
(Centrifuge CN-2200 MRC). The fluorescence intensity of the supernatant was then measured at $750 \mathrm{~nm}$.

\section{Conjugation of peanut agglutinin and $\alpha$ CEA to NIR fluorescent IO-HSA nanoparticles}

Covalent conjugation of peanut agglutinin and $\alpha$ CEA tumortargeting ligands to NIR fluorescent IO-HSA nanoparticles was accomplished in two steps as follows:

- Activation of the NIR fluorescent IO-HSA nanoparticles by NHS-PEG-NHS spacer arm molecules in excess. Briefly, $0.5 \mathrm{~mL}$ of NHS-PEG-NHS aqueous solution $(10 \mathrm{mg} / \mathrm{mL})$ was added to $5 \mathrm{~mL}$ of NIR fluorescent IO-HSA nanoparticles dispersed in PBS $(1 \mathrm{mg} / \mathrm{mL}$, $\mathrm{pH}$ 7.4). The reaction mixture was then shaken at $4^{\circ} \mathrm{C}$ for 15 minutes. The NIR fluorescent IO-HSA-PEGNHS nanoparticles obtained were then washed with PBS from nonmagnetic waste using the magnetic gradient column.

- Covalent conjugation of peanut agglutinin to the NIR fluorescent IO-HSA-PEG-NHS nanoparticles. Briefly, $40 \mu \mathrm{L}$ of peanut agglutinin solution $(4 \mathrm{mg} / \mathrm{mL})$ were added to $500 \mathrm{~mL}$ of the NIR fluorescent IO-HSA-PEGNHS nanoparticles dispersed in PBS $(2 \mathrm{mg} / \mathrm{mL}, \mathrm{pH} 7.4$, $[$ Nanoparticles $] /[$ peanut agglutinin $]=20(\mathrm{w} / \mathrm{w}))$. The reaction mixture was then shaken at $4^{\circ} \mathrm{C}$ for 20 minutes. Residual NHS groups were then blocked by addition of glycine $(1 \% \mathrm{w} / \mathrm{v})$. The mixture was then shaken for a further 30 minutes. The peanut agglutinin-conjugated NIR fluorescent IO-HSA nanoparticles obtained were then washed with PBS from nonmagnetic waste using magnetic gradient columns.

FITC-PNA or $\alpha$ CEA antibodies were conjugated with the NIR fluorescent IO-HSA nanoparticles via a similar procedure substituting the peanut agglutinin with the FITCPNA or $\alpha C E A$. The concentration of bound peanut agglutinin $(2.1 \pm 0.3 \mu \mathrm{g} / \mathrm{mg}$ nanoparticles) was determined with FITCPNA via a calibration curve obtained from known concentrations of PNA-FITC. The concentration of bound $\alpha$ CEA antibodies (1.8 $\pm 0.2 \mu \mathrm{g} / \mathrm{mg}$ nanoparticles) was determined using a mouse IgG enzyme-linked immunosorbent assay kit (Biotest Ltd, Kfar Saba, Israel).

\section{Characterization of functional NIR fluorescent IO-HSA nanoparticles}

Low-resolution transmission electron microscopy pictures were obtained using a FEI Tecnai C2 Biotwin electron microscope with an accelerating voltage of $120 \mathrm{kV}$. Samples for transmission electron microscopy were prepared by placing a drop of a diluted sample on a 400 mesh carboncoated copper grid. The average size and size distribution of the dry particles were determined by measuring the diameter of more than 100 particles with AnalySIS Auto image analysis software (Soft Imaging System GmbH, Munster, Germany).

The hydrodynamic diameter and size distribution of the nanoparticles dispersed in aqueous phase were measured by dynamic light scattering with photon cross-correlation spectroscopy (Nanophox particle analyzer, Sympatec GmbH, Clausthal- Zellerfeld, Germany).

Fluorescence measurements of the FITC-PNA were performed using a Tecan SpectraFluor Plus multiplate reader (Neotec Scientific Instruments, Kfar Saba, Israel) with an excitation wavelength of $485 \pm 10 \mathrm{~nm}$, an emission wavelength of $535 \pm 10 \mathrm{~nm}$, an integration time of $40 \mu \mathrm{sec}$, and gain set to 70 .

Fluorescence spectra of the NIR fluorescent IO and IOHSA nanoparticles were recorded using a spectrofluorometer (Cary Eclipse; Agilent Technologies Inc, Santa Clara, CA). Excitation and emission slits were fixed at $5 \mathrm{~nm}$, and $\lambda_{\text {ex }}$ was set at $750 \mathrm{~nm}$.

\section{Measurement of CANIR concentration entrapped in NIR fluorescent IO-HSA nanoparticles}

A calibration curve for CANIR was obtained by plotting the integral of the absorbance of a series of standard aqueous solutions of CANIR $(0.5-10 \mu \mathrm{g} / \mathrm{mL})$ against concentration. The absorbance spectrum of a $1 \mathrm{mg} / \mathrm{mL}$ aqueous nanoparticle dispersion was recorded, and the integral of absorbance at 70-890 nm was determined. The concentration of the entrapped dye according to the calibration curve was estimated to be $1.25 \mu \mathrm{g}$ dye per mg particles.

\section{Photostability of NIR fluorescent IO-HSA nanoparticles}

A solution of CANIR in PBS $(0.57 \mathrm{nM}, 500 \mu \mathrm{L})$ and an aqueous dispersion of NIR fluorescent IO-HSA nanoparticles $(0.25 \mathrm{mg} / \mathrm{mL})$ were prepared. These solutions showed similar fluorescence intensity at an excitation wavelength of $800 \mathrm{~nm}$ and an emission wavelength of $830 \mathrm{~nm}$. The excitation slit was opened to $20 \mathrm{~nm}$ and the emission slit was opened to $5 \mathrm{~nm}$. Each of the samples was illuminated continuously, and the fluorescence intensity was measured 
over a period of 20 minutes using a Cary Eclipse fluorescence spectrophotometer (Agilent Technologies Inc). Intensity values were normalized for comparison.

\section{Fluorescence imaging of NIR fluorescent IO-HSA nanoparticles}

Fluorescent images were acquired using a Maestro II in vivo fluorescence imaging system (Cambridge Research and Instrumentation Inc, Woburn, MA). A NIR excitation/ emission filter set was used for our experiments $\left(\lambda_{\text {ex }}\right.$ $\left.770-700 \mathrm{~nm}, \lambda_{\mathrm{em}}>790 \mathrm{~nm}\right)$. The liquid crystal tunable filter was programmed to acquire image cubes from $\lambda=790 \mathrm{~nm}$ to $860 \mathrm{~nm}$ with an increment of $10 \mathrm{~nm}$ per image.

\section{Cell lines}

Human LS174T and SW480 colorectal adenocarcinoma cell lines were used for the following experiments. The LS174T cell line was maintained in Eagle's minimum essential medium supplemented with heat-inactivated fetal bovine serum $10 \%$, penicillin $100 \mathrm{IU} / \mathrm{mL}$, streptomycin $100 \mu \mathrm{g} / \mathrm{mL}$, and l-glutamine $2 \mathrm{mM}$. The SW480 cell line was maintained in Dulbecco's minimum essential medium supplemented with fetal bovine serum $10 \%$, penicillin $100 \mathrm{IU} / \mathrm{mL}$, streptomycin $100 \mu \mathrm{g} / \mathrm{mL}$, and l-glutamine $2 \mathrm{mM}$.

\section{CAM grafting procedure}

Tumor cells were grafted on the CAM in the manner described in the literature. ${ }^{32}$ Briefly, fertile chicken eggs were incubated at $37^{\circ} \mathrm{C}$ and $60 \%-70 \%$ humidity in a forceddraft incubator. After 3 days of incubation, an artificial air sac was created above the CAM. On day 8 of incubation, a window was opened into the shell and the CAM exposed. Tumor cells were collected by trypsinization, washed with culture medium and pelleted by gentle centrifugation. After removing the medium, $5 \times 10^{6}$ cells were resuspended in $30 \mu \mathrm{L}$ ice-cold Matrigel and inoculated on the CAM at the site of the blood vessels. The eggs were then sealed and placed back in the incubator. On day 6 after grafting (day 14 of incubation), the tumor size ranged from $3 \mathrm{~mm}$ to $5 \mathrm{~mm}$ in diameter, with visible neoangiogenesis.

\section{Detection of human colon tumor by NIR fluorescent bioactive IO-HSA nanoparticles \\ CAM tumor detection}

Chicken embryos with six-day-old tumors (LS174T and SW480 cell lines) implanted on the CAM (triplets for each set of experiment) were treated with peanut agglutinin or $\alpha C E A$-conjugated NIR fluorescent IO-HSA nanoparticles dispersed in PBS $(40 \mu \mathrm{L}, 0.7 \mathrm{mg} / \mathrm{mL})$ and with glycineconjugated nanoparticles (as controls). Nonpathological CAM was similarly treated with the nanoparticles as an additional control. Twenty minutes later, the tumors and the nonpathological CAM were removed from the eggs and washed with PBS, and then spread on a nonreflective black background for observation using the Maestro ${ }^{\mathrm{TM}}$ in vivo imaging system. The experiment was repeated four times. Fluorescence intensity measurements were performed using Image J software. ${ }^{32}$

\section{In vivo orthotopic mouse tumor detection}

Experiments were performed by Harlan Biotech (Rehovot, Israel) according to the protocols of the Israeli National Council for Animal Experiments. A dozen athymic nude mice (a quartet for each set of experiments) with implanted 2-week old lumen-facing LS174T tumors were anesthetized and treated with peanut agglutinin or $\alpha C E A$-conjugated NIR fluorescent IO-HSA nanoparticles dispersed in PBS $(0.2 \%, 200 \mu \mathrm{L})$, via the anus, using the guidance of mini-colonoscopy (Medigus Ltd, Omer, Israel). Twenty minutes later, the colons were washed extensively with PBS and allowed to recover for 4 hours. The mice were then sacrificed and the colons were removed. Each colon was spread on a clear film, and imaging was performed using the Odyssey ${ }^{\circledR}$ infrared imaging system (Li-Cor Biosciences, Lincoln, NE), with an excitation wavelength of $780 \mathrm{~nm}$ and an emission wavelength of $800 \mathrm{~nm}$.

The colonoscopy procedure may wound the colon at the penetration location where the fluorescent nanoparticles are sprayed, due to the size of the colonoscope. Therefore, we used a mini-colonoscope especially made for rodents, although its size is still large relative to the size of the mice. This particular colonoscope was chosen because of its special features that include a spray catheter and a camera.

\section{Results and discussion CANIR dye}

The cyanine dye chosen for use throughout this work is a previously synthesized carboxylic acid derivative of commercially available IR-783, as described in Figure $1 .{ }^{33}$ This dye is a dark green powder obtained in high yield (90\%) and $100 \%$ purity as shown by elemental analysis, mass spectroscopy, and ${ }^{1} \mathrm{H}$ nuclear magnetic resonance. Carboxylic acid functionality was used for covalent attachment of the dye to the primary amino groups of gelatin via the carbodiimide activation method. ${ }^{25} \mathrm{CANIR}$-conjugated gelatin 
was then used for the preparation of the NIR fluorescent IO nanoparticles.

\section{NIR fluorescent IO-HSA nanoparticles}

NIR fluorescent IO nanoparticles were prepared by nucleation followed by controlled growth of thin IO films onto CANIR-gelatin-IO nuclei. These fluorescent IO nanoparticles were then coated using HSA aqueous solution containing the CANIR dye via a precipitation process at high temperature, as described in the experimental section. ${ }^{25,31}$ NIR dyes such as indocyanine green and other structurally related cyanine dyes have been shown to have high affinity for albumin. ${ }^{34}$ Molecular modeling illustrates that albumin has two binding sites responsible for the strong physical binding of NIR cyanine dyes to albumin. ${ }^{26,35,36}$ This strong physical binding of NIR polymethine dyes to albumin was exploited to form the fluorescent coating of the present NIR fluorescent IOHSA nanoparticles.

A second HSA coating was performed after removal of excess dye in the absence of additional CANIR. This was in order to ensure that the dye is present within the HSA coating and not on the particle surface, because this could lead to nanoparticle aggregation resulting from the high affinity of the dye for HSA.

The main advantage of these NIR fluorescent IO-HSA nanoparticles is that the fluorescent dye is encapsulated within the nanoparticles and not surface-bound, as in most cited prepared fluorescent nanoparticles. ${ }^{37-39}$ Therefore, the surface properties such as surface functional composition and zeta potential, of the nanoparticles are retained.

The measured diameter and size distribution of the NIR fluorescent IO-HSA nanoparticles are shown in Figure 2.
A transmission electron microscopic image of the NIR fluorescent IO-HSA nanoparticles demonstrates that they have a dry diameter of $15.0 \pm 4 \mathrm{~nm}$ and are stable against aggregation (Figure 2A). In contrast with the transmission electron microscopic image, the size of the NIR fluorescent IO-HSA nanoparticles dispersed in a continuous aqueous phase, as determined by dynamic light scattering, was $67 \pm 9 \mathrm{~nm}$ (Figure 2B). While transmission electron microscopic measures the dry diameter of the particles, dynamic light scattering measures the hydrodynamic diameter which takes into account the water molecules adsorbed onto the particle surface. This difference explains the higher diameter of the nanoparticles measured by dynamic light scattering compared with those measured by transmission electron microscopic.

It should be noted that the dry diameter of the noncoated IO nanoparticles was similar to or slightly lower than that of the IO-HSA nanoparticles. On the other hand, the hydrodynamic diameter of the noncoated IO particles was significantly higher than that of IO-HSA $(83 \pm 12$ and $67 \pm 9 \mathrm{~nm}$, respectively, as shown in Figure 2B). The decrease in the hydrodynamic diameter of these coated HSA nanoparticles may be explained by the decreased thickness of the adsorbed layer of water molecules as a consequence of its relatively higher surface hydrophobicity compared with the noncoated IO.

\section{Fluorescence measurements}

The absorbance and emission spectra of the NIR fluorescent IO-HSA nanoparticles and free CANIR in PBS are shown in Figure 3. The maximum absorption of free CANIR and NIR fluorescent IO-HSA nanoparticles occurs at approximately
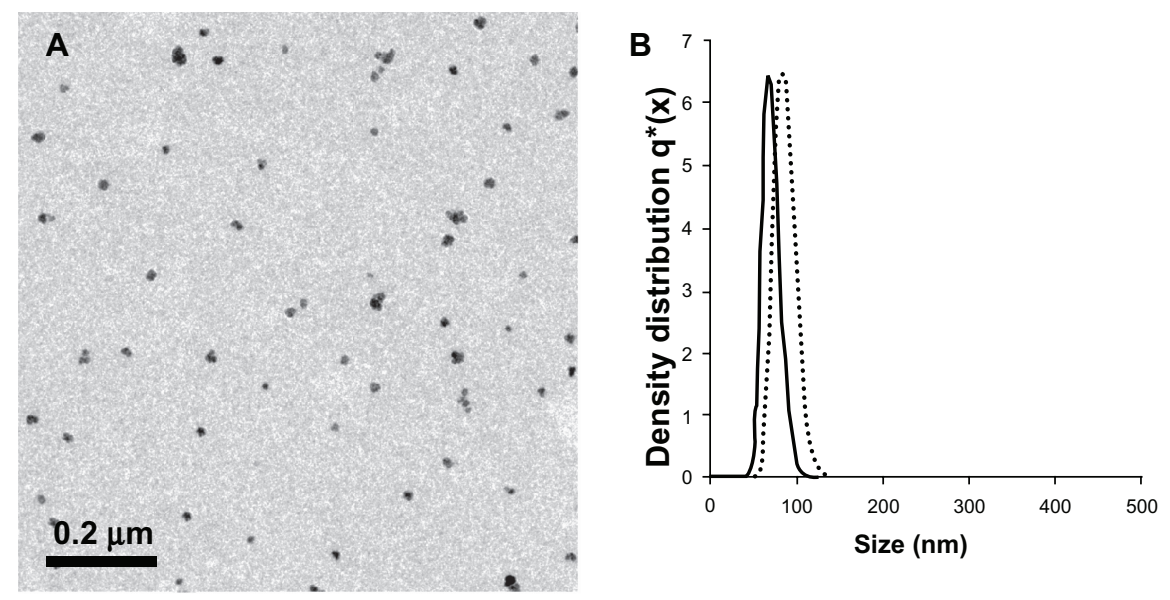

Figure 2 (A) Transmission electron photomicrograph of the near-infrared fluorescent iron oxide-human serum albumin nanoparticles. (B) Histograms demonstrating the diameter and size distribution of the noncoated (dots) and coated (solid line) near-infrared fluorescent iron oxide nanoparticles dispersed in water. 


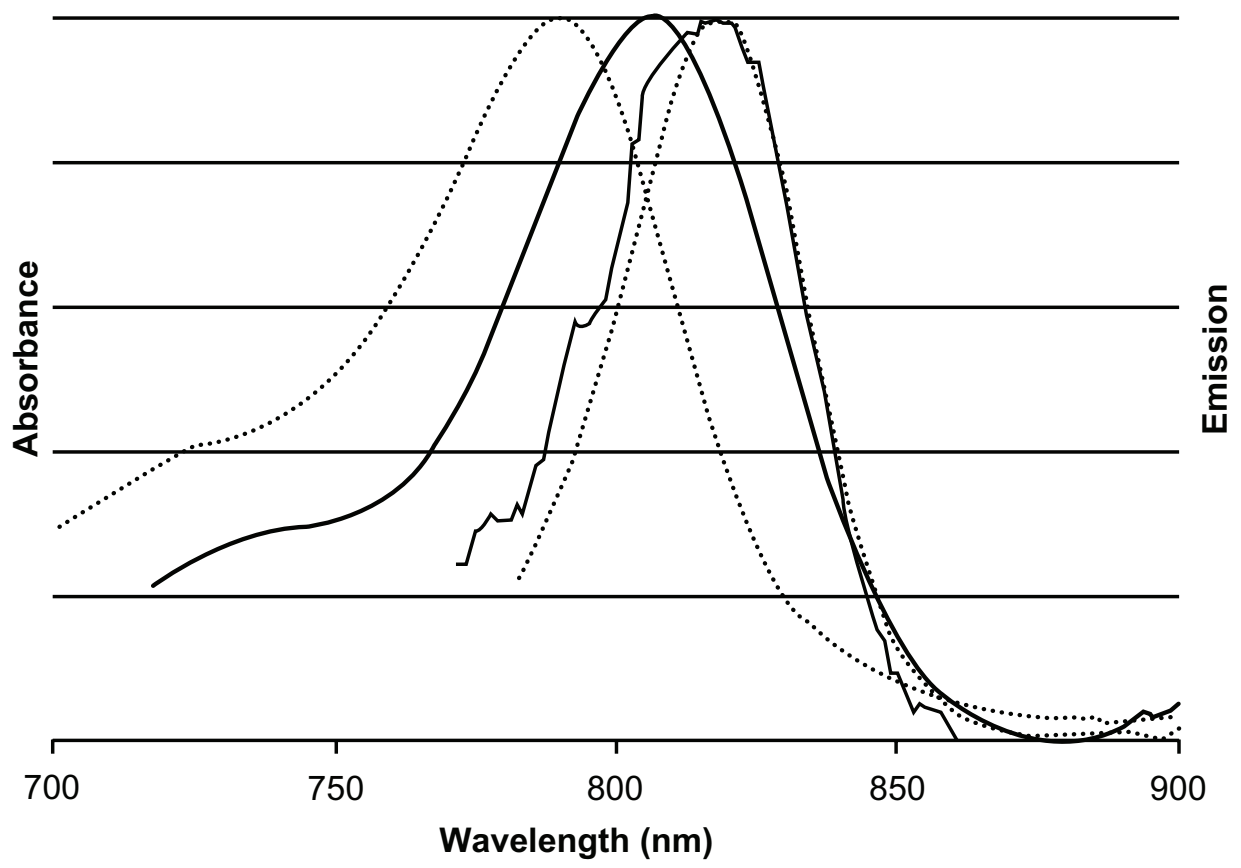

Figure 3 Normalized absorbance and emission spectra of free cyanine near-infrared fluorescent dye (dotted lines) and near-infrared fluorescent iron oxide-human serum albumin nanoparticles (solid lines).

Notes: Maximum absorption of free cyanine near-infrared fluorescent dye and near-infrared fluorescent iron oxide-human serum albumin nanoparticles occur at approximately $790 \mathrm{~nm}$ and $810 \mathrm{~nm}$, respectively. Maximum fluorescence emission intensity of cyanine near-infrared fluorescent dye and near-infrared fluorescent iron oxide-human serum albumin nanoparticles occurs at the same wavelength of approximately $818 \mathrm{~nm}$.

$790 \mathrm{~nm}$ and $804 \mathrm{~nm}$, respectively. The maximum fluorescence emission intensity of both the free dye and the dye-conjugated nanoparticles occurs at $818 \mathrm{~nm}$. The red-shift in the absorbance spectrum of the NIR fluorescent IO-HSA nanoparticles compared with free CANIR dye is probably due to its physical binding to HSA, that places the dye in a more hydrophobic environment and affects the dipole moment of the dye..$^{40}$

Three types of NIR fluorescent IO-HSA nanoparticles were synthesized for comparison, ie, IO containing dyed gelatin in the core and a nondyed HSA shell, IO containing nondyed gelatin in the core and a dyed HSA shell, and IO containing dyed gelatin in the core as well as a dyed HSA shell. A comparison of the fluorescence intensity of each type shows that the nanoparticles composed of dye within the core as well as within the shell (nanoparticles of type 3) possess the highest fluorescence intensity (Figure 4). The concentration of the dye encapsulated within each of the compared NIR fluorescent nanoparticles was quantified using a calibration curve measuring the integral of the absorbance spectra of different concentrations of free dye in aqueous solution (700-890 $\mathrm{nm})$. The concentration of the CANIR dye encapsulated within the NIR fluorescent nanoparticles, composed of dyed gelatin in the core as well as a dyed HSA shell, was estimated to be $1.25 \mu \mathrm{g}$ per mg of nanoparticles.
Approximately half the amount of the dye was found to be in each of the other two types of fluorescent nanoparticles. However, in spite of the fact that the concentration of the CANIR dye in the nanoparticles composed of dyed gelatin core (type 1) and those composed of dyed HSA shell (type 2) was the same, the fluorescence intensity of the latter was

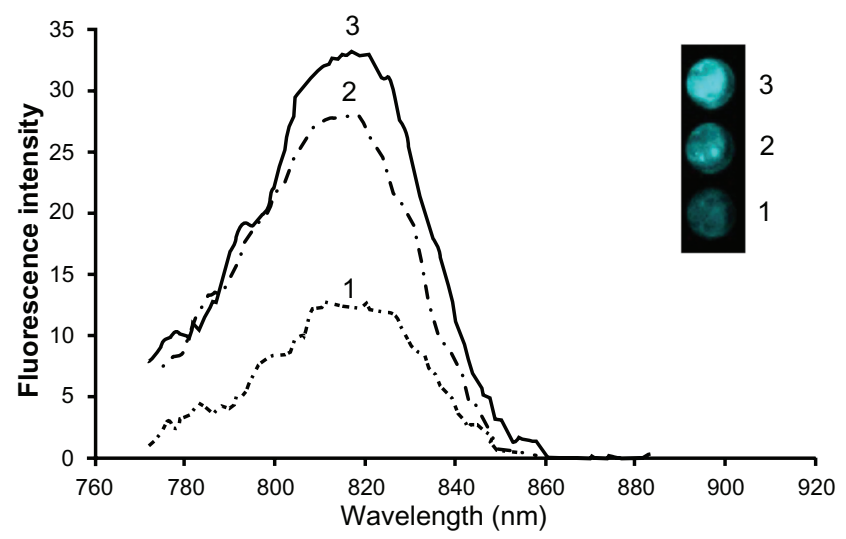

Figure 4 Comparison of fluorescence intensities of equal concentrations of three types of near-infrared fluorescent iron oxide-human serum albumin nanoparticles composed of: iron oxide containing dyed gelatin in the core and a non-dyed human serum albumin shell; iron oxide containing non-dyed gelatin in the core and a dyed human serum albumin shell; iron oxide containing dyed gelatin in the core as well as in a dyed human serum albumin shell.

Notes: The highest fluorescence intensity was shown by particles containing dye within both the core and the shell (type 3). The inset shows a fluorescence image of equal concentrations of each type of particle dispersed in phosphate-buffered saline solution. 
significantly higher than that of the former, as shown in Figure 4. Because the fluorescence intensity of the nanoparticles composed of a dyed gelatin core and dyed HSA shell was the highest, we chose these nanoparticles for continuation of the present study.

Leakage of the encapsulated CANIR dye of these nanoparticles into the aqueous continuous phase in the absence or presence of $4 \%$ soluble HSA was not detected following ultrafiltration, as described in the experimental section, confirming the strong physical interaction of CANIR to HSA, as already known from the literature..$^{26,35}$

\section{Photostability}

To examine the photostability of NIR fluorescent nanoparticles, we performed photobleaching experiments for free CANIR dye and CANIR encapsulated within the IO-HSA nanoparticles. Samples of each were illuminated at $800 \mathrm{~nm}$, and their fluorescence intensities were measured. During illumination, the fluorescence intensity of the NIR fluorescent IO-HSA nanoparticles remained almost unaltered while that of free CANIR decreased significantly $(\mathrm{t}=20$ minutes, $28 \%$, $P<0.001$ ), as shown in Figure 5.

Photobleaching is the irreversible light-induced destruction of the fluorophore, affected by factors such as oxygen, oxidizing or reducing agents, temperature, exposure time, and illumination levels. ${ }^{41}$ Figure 5 demonstrates that encapsulation of CANIR within the nanoparticles significantly reduced the photobleaching. Encapsulation of the dye probably protects the dye against reactive oxygen species, thereby reducing photobleaching. ${ }^{23,41}$

\section{Tumor growth on CAM}

A chicken embryo CAM model was used in this work for testing specific tumor detection by both nonbioactive and bioactive peanut agglutinin-conjugated and $\alpha \mathrm{CEA}-$ conjugated NIR fluorescent IO-HSA nanoparticles. LS174T and SW480 cell lines evaluated in this study formed solid tumors $3-5 \mathrm{~mm}$ in diameter within 6 days. Figure 6 shows a typical LS174T cell line-derived tumor delimited by a plastic ring on a chicken CAM. A similar tumor was also observed for the SW480 cell line.

\section{Optical detection of human colon tumor} Chicken embryo CAM model

Peanut agglutinin and $\alpha$ CEA were used for targeting of colon carcinomas. Peanut agglutinin binds to the terminal sugar, $\beta$-D-galactosyl-(1-3)-N-acetyl-D-galactosamine, of the Thomsen-Friedenreich antigen that is upregulated on the mucosal side of various colorectal cancer cell lines, such as LS174T, in comparison with noncancerous cells. ${ }^{42}$ CEA, a highly glycosylated glycoprotein, is highly expressed in most human carcinomas and therefore frequently used as a marker. ${ }^{28}$ Peanut agglutinin, $\alpha$ CEA, or glycine as a control were covalently conjugated with the NIR fluorescent IO-HSA nanoparticles via a $3 \mathrm{kDa}$ PEG spacer, as described in the experimental section. The tumors as well

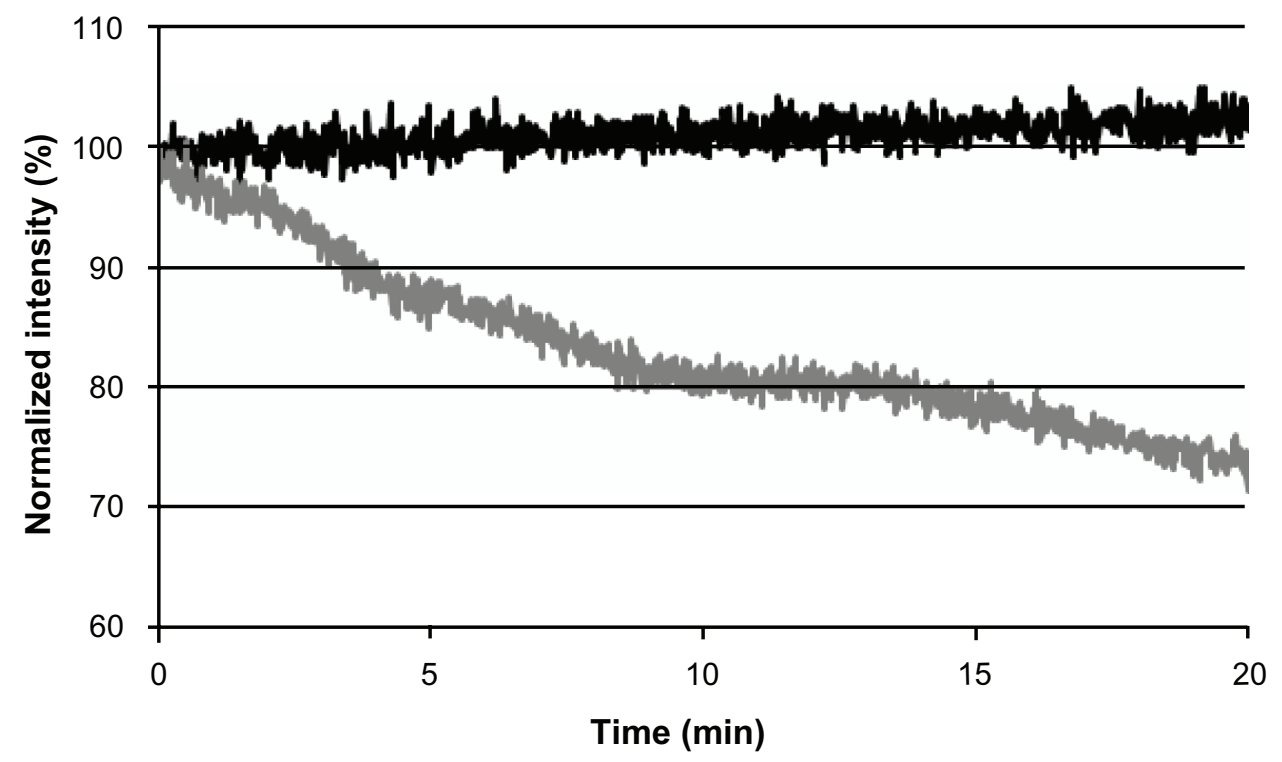

Figure 5 Photostability of the near-infrared fluorescent iron oxide-human serum albumin nanoparticles (black line) and free cyanine near-infrared fluorescent dye (gray line) as a function of time.

Note: Samples of cyanine near-infrared fluorescent dye containing iron oxide-human serum albumin nanoparticles and free cyanine near-infrared fluorescent dye were illuminated with a Xenon flash lamp for 20 minutes as described in the experimental section. 


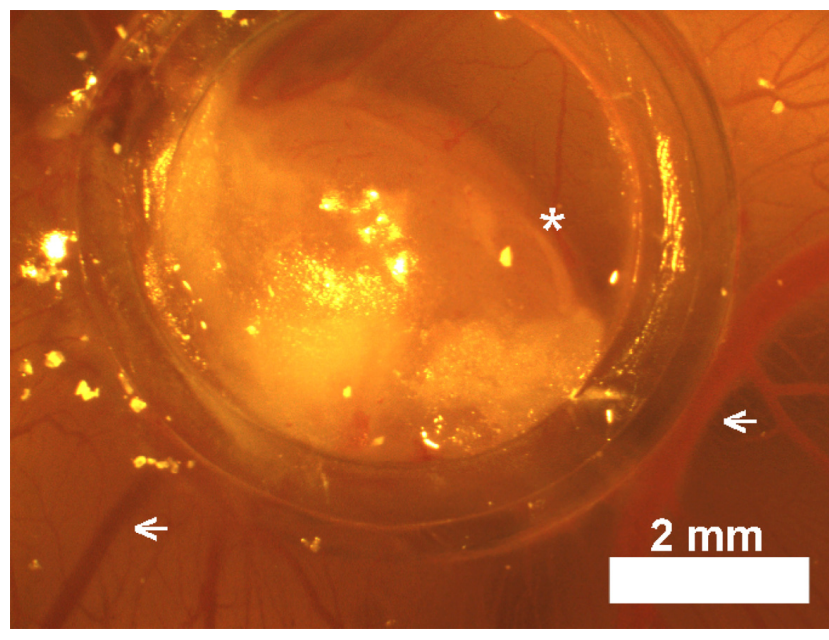

Figure 6 Light photograph of a LSI74T tumor delimited by a plastic ring on chicken chorioallantoic membrane in situ in the egg.

Note: Suspensions of $5 \times 10^{6}$ LSI74T cells in Matrige ${ }^{\circledR}$ formed compact structures (asterisks) 7 days after transplantation that apparently attracted host blood vessels (arrows).

as the nonpathological CAM were treated topically with a dispersion of the various nanoparticles in PBS, and 20 minutes later were washed with PBS. The tumors were removed and fluorescence imaging was performed. Figure 7 shows that the glycine-conjugated (control) nanoparticles exhibited a relatively low fluorescence signal. In contrast, when the tumors were treated with $\alpha \mathrm{CEA}$-conjugated or peanut agglutinin-conjugated nanoparticles, the fluorescence intensity of the tumors was about five and seven times, respectively, higher than that of the control nanoparticles (Figure 7B). It should be noted that the nonpathological CAM remained unlabeled by the glycine, $\alpha \mathrm{CEA}$, or peanut agglutinin-conjugated nanoparticles. The fluorescence
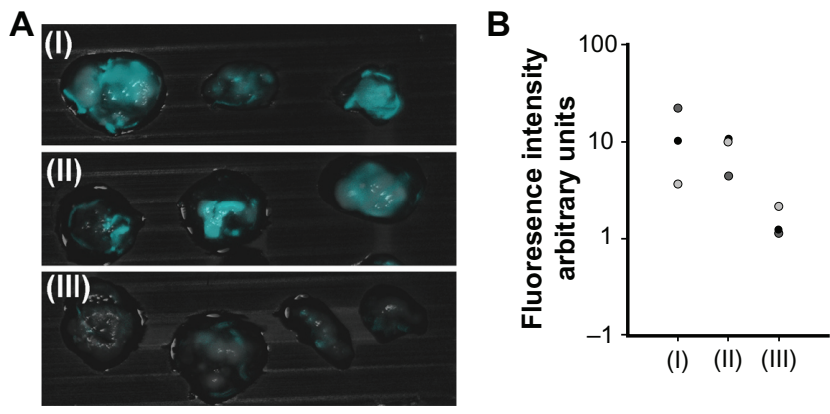

Figure 7 Fluorescence imaging of tumors on chicken embryo chorioallantoic membrane. (A) Merged fluorescent and bright light images of a typical experiment involving a LSI74T tumor cell line implanted in chicken embryo chorioallantoic membrane treated with peanut agglutinin-conjugated (I), anticarcinoembryonic antigen antibody-conjugated (II), and glycine (III)-conjugated iron oxide-human serum albumin near-infrared fluorescent nanoparticles. (B) Quantification of the fluorescence intensity of the tumors averaged over the surface area as calculated by Imagej software.

Notes: Vertical axis is logarithmically scaled. This experiment was repeated three more times with similar results. intensity of the nontreated tumors was also negligible, indicating that there is no significant autofluorescence of these tumors in the NIR range.

In addition to the LS174T cell line, we studied the interaction of the bioactive nanoparticles with the control SW480 cell line. According to the literature, CEA and Thomsen-Friedenreich antigens are expressed to a much lower extent (at least $10^{3}$ times) in the SW480 cell line than in the LS174T cell line. ${ }^{42,43}$ Indeed, Figure 8 shows that the fluorescence intensity of the LS174T tumor cell line treated with peanut agglutinin-conjugated or $\alpha \mathrm{CEA}$-conjugated fluorescent IO-HSA nanoparticles is 10-30 times higher than that observed for the SW480 cell line (Figure 8B). These results indicate that the target molecules maintain their activity when conjugated with the nanoparticles. This targeting activity is seen through the significant increase in fluorescence intensity of the LS174T tumor labeled by the bioactivated, conjugated nanoparticles over the controlled particles and the control SW480 tumor. The significant increase in tumor fluorescence could be attributed to interaction of the bioactive, conjugated nanoparticles with tumor cell surface receptors.

\section{Orthotopic mouse colon model}

In vivo labeling of colonic tumors was performed using an orthotopic mouse model, with colonic tumors originating from LS174T cells injected into the colon wall 2 weeks before the experiment. Mice were anesthetized and treated with peanut agglutinin-conjugated or $\alpha \mathrm{CEA}$ conjugated nanoparticles dispersed in PBS, via the anus, as described in the experimental section. The mouse colons
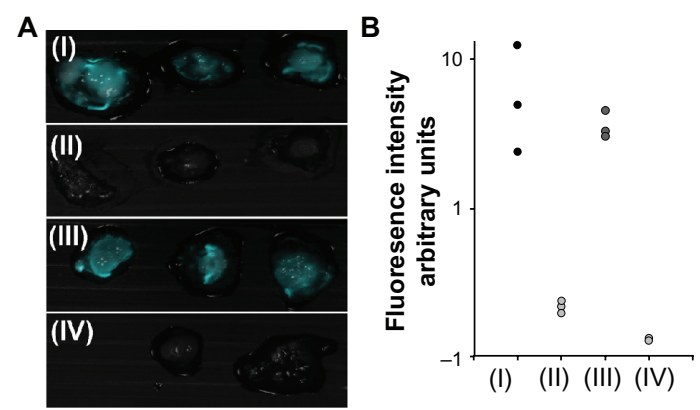

Figure 8 Fluorescence imaging of tumors on chicken embryo chorioallantoic membrane. (A) Merged fluorescent and bright light images of a typical experiment involving peanut agglutinin-conjugated or anticarcinoembryonic antigen antibodyconjugated iron oxide-human serum albumin NIR fluorescent nanoparticles topically administered to LSI74T (I and III, respectively) and SW480 (II and IV, respectively) tumor cell lines implanted on chicken embryo chorioallantoic membrane. (B) Quantification of fluorescence intensity of tumors averaged over the surface area, as calculated by Imagej software.

Notes: Vertical axis is logarithmically scaled. This experiment was repeated three more times with similar results. 

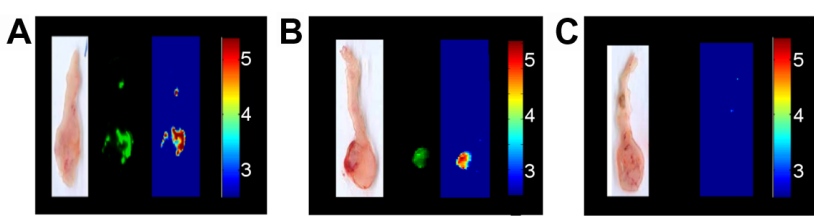

Figure 9 Typical experiment showing a mouse colon treated with (A) peanut agglutinin-conjugated and (B) anticarcinoembryonic antigen antibody-conjugated iron oxide-human serum albumin near-infrared fluorescent nanoparticles. Each set of experiments includes a color photograph, a fluorescent image, and a logarithmically scaled fluorescent image. (C) Untreated tumor indicating a negligible level of tumor autofluorescence. (B and C) Bioactive, conjugated fluorescent iron oxide-human serum albumin nanoparticles selectively label the LSI74T tumors and leave the surrounding nonpathological tissue relatively unlabeled, with a signal-to-background ratio of $\mathrm{I}: \mathrm{I} \times \mathrm{e}^{25}$.

Note: This experiment was repeated three more times with similar results.

were then removed and washed extensively. The fluorescence intensity of the implanted tumors was 3-13 times higher than that of the surrounding nonpathological tissue (Figure 9A and B). Under the same experimental conditions, the autofluorescence signal of the nontreated tumor cell lines was negligible (Figure 9C). An additional small fluorescent signal appears in close proximity to the anus, as shown in Figure 9A. It is possible that this fluorescent signal indeed indicates a small cancerous growth which is not visible to the eye. Another possibility is that the colonoscopy procedure wounded the colon at the sprayed site, so that a small portion of the fluorescent nanoparticles got trapped inside the wounded location and could not be washed off. It should be noted that such wounding would probably not occur in human beings.

Specifically labeled colon tumors in both chicken and mouse models suggest that peanut agglutinin-conjugated or $\alpha C E A-c o n j u g a t e d$ NIR fluorescent IO-HSA nanoparticles could be excellent candidates for early detection of tumors in the human colon.

\section{Conclusion}

In this study, we prepared IO-HSA NIR fluorescent nanoparticles for detection of colon cancers. These NIR fluorescent nanoparticles may prove to be very useful for in vivo tumor detection due to the low NIR autofluorescence of body tissues and relatively deep penetration into biomatrices. We have demonstrated that encapsulation of NIR dye significantly stabilizes the dye against photobleaching. We have also established in chicken embryo and mouse models that peanut agglutinin-conjugated and $\alpha \mathrm{CEA}$-conjugated NIR fluorescent IO-HSA nanoparticles maintain their activity and specifically label cancerous tissue, leaving surrounding nonpathological tissue unlabeled. In addition, under our study conditions, no significant autofluorescence was observed.
Therefore, these new bioactive, conjugated IO-HSA NIR fluorescent nanoparticles may serve as an efficient probe for specific optical visualization of colon cancer using NIR fluorescence endoscopic systems. ${ }^{2,7}$

In future work, we plan to extend this study to other tumortargeting ligands including antibodies, proteins, and small peptides, such as epidermal growth factor, tumor-associated glycoprotein-72 monoclonal antibodies, and TRAIL. ${ }^{44,45}$ Furthermore, cancer drugs such as paclitaxel and doxorubicin may also be encapsulated within these bioactive IO-HSA NIR fluorescent nanoparticles, consequently enabling the use of these particles for both diagnosis and therapy.

\section{Acknowledgments}

This study was supported by the Israeli Ministry of Commerce and Industry (BMP Consortium of Biomedical Photonics). The authors thank Igor Grinberg for assistance with the chicken embryo model, Harlan Biotech for assistance with the in vivo mice experiments, and Ronen Yehuda for assistance with the fluorescent images.

\section{Disclosure}

The authors report no conflicts of interest in this work.

\section{References}

1. Detsky AS. Screening for colon cancer - can we afford colonoscopy? N Engl J Med. 2001;345(8):607-608.

2. Kondepati VR, Keese M, Mueller R, Manegold BC, Backhaus J. Application of near-infrared spectroscopy for the diagnosis of colorectal cancer in resected human tissue specimens. Vib Spectrosc. 2007;44(2): 236-242.

3. Zeng H, Weiss A, Cline R, MacAulay CE. Real-time endoscopic fluorescence imaging for early cancer detection in the gastrointestinal tract. Bioimaging. 1998;6(4):151-165.

4. Altınoğlu Eİ, Adair JH. Near-infrared imaging with nanoparticles. Wiley Interdiscip Rev Nanomed Nanobiotechnol. 2010;2(5):461-477.

5. Jiang S, Gnanasammandhan MK, Zhang Y. Optical imaging-guided cancer therapy with fluorescent nanoparticles. JR Soc Interface. 2010; 7(42):3-18.

6. Santra S, Malhotra A. Fluorescent nanoparticle probes for imaging of cancer. Wiley Interdiscip Rev Nanomed Nanobiotechnol. 2011;3(5): 501-510.

7. Hsiung PL, Hardy J, Friedland S, et al. Detection of colonic dysplasia in vivo using a targeted heptapeptide and confocal microendoscopy. Nat Med. 2008;14(4):454-458.

8. Altınoğlu EI, Russin TJ, Kaiser JM, et al. Near-infrared emitting fluorophore-doped calcium phosphate nanoparticles for in vivo imaging of human breast cancer. ACS Nano. 2008;2(10):2075-2084.

9. Chen W, Jarzyna PA, van Tilborg GAF, et al. RGD peptide functionalized and reconstituted high-density lipoprotein nanoparticles as a versatile and multimodal tumor targeting molecular imaging probe. FASEB J. 2010;24(6):1689-1699.

10. Le Guevel X, Hotzer B, Jung G, Schneider M. NIR-emitting fluorescent gold nanoclusters doped in silica nanoparticles. J Mater Chem. 2011; 21(9):2974-2981.

11. He X, Wang K, Cheng Z. In vivo near-infrared fluorescence imaging of cancer with nanoparticle-based probes. Wiley Interdiscip Rev Nanomed Nanobiotechnol. 2010;2(4):349-366. 
12. Luo S, Zhang E, Su Y, Cheng T, Shi C. A review of NIR dyes in cancer targeting and imaging. Biomaterials. 2011;32(29):7127-7138.

13. Sharma P, Brown S, Walter G, Santra S, Moudgil B. Nanoparticles for bioimaging. Adv Colloid Interface Sci. 2006;123-126:471-485.

14. Chen J, Corbin IR, Li H, Cao W, Glickson JD, Zheng G. Ligand conjugated low-density lipoprotein nanoparticles for enhanced optical cancer imaging in vivo. J Am Chem Soc. 2007;129(18):5798-5799.

15. Cho K, Wang X, Nie S, Shin DM. Therapeutic nanoparticles for drug delivery in cancer. Clin Cancer Res. 2008;14(5):1310-1316.

16. Morton CL, Houghton PJ. Establishment of human tumor xenografts in immunodeficient mice. Nat Protoc. 2007;2(2):247-250.

17. Durupt F, Koppers-Lalic D, Balme B, et al. The chicken chorioallantoic membrane tumor assay as model for qualitative testing of oncolytic adenoviruses. Cancer Gene Ther. 2012;19(1):58-68.

18. Xie J, Liu G, Eden HS, Ai H, Chen X. Surface-engineered magnetic nanoparticle platforms for cancer imaging and therapy. Acc Chem Res. 2011;44(10):883-892.

19. Banerjee R, Katsenovich Y, Lagos L, McIintosh M, Zhang X, Li CZ. Nanomedicine: magnetic nanoparticles and their biomedical applications. Curr Med Chem. 2010;17(27):3120-3141.

20. Hergt R, Dutz S, Zeisberger M. Validity limits of the Néel relaxation model of magnetic nanoparticles for hyperthermia. Nanotechnology. 2010;21:015706.

21. Margel S, Burdygin I, Reznikov V, et al. Functional nanoparticles: synthesis and biological applications. Recent Res Dev Polym Sci. 1997; 1:51-78.

22. van Buul GM, Kotek G, Wielopolski PA, et al. Clinically translatable cell tracking and quantification by MRI in cartilage repair using superparamagnetic iron oxides. PloS One. 2011;6(2):e17001.

23. Perlstein B, Lublin-Tennenbaum T, Marom I, Margel S. Synthesis and characterization of functionalized magnetic maghemite nanoparticles with fluorescent probe capabilities for biological applications. J Biomed Mater Res B Appl Biomater. 2010;92(2):353-360.

24. Ziv O, Avtalion RR, Margel S. Immunogenicity of bioactive magnetic nanoparticles: natural and acquired antibodies. J Biomed Mater Res A. 2008;85(4):1011-1021.

25. Corem-Salkmon E, Ram Z, Daniels D, et al. Convection-enhanced delivery of methotrexate-loaded maghemite nanoparticles. Int $J$ Nanomedicine. 2011;6:1595-1602.

26. Berezin MY, Lee H, Akers W, Nikiforovich G, Achilefu S. Ratiometric analysis of fluorescence lifetime for probing binding sites in albumin with near-infrared fluorescent molecular probes. Photochem Photobiol. 2007;83(6):1371-1378.

27. Sakuma S, Higashino H, Oshitani H, et al. Essence of affinity and specificity of peanut agglutinin-immobilized fluorescent nanospheres with surface poly(N-vinylacetamide) chains for colorectal cancer. Eur J Pharm Biopharm. 2011;79(3):537-543.

28. Tsai H, Chang C, Li Y, Chu W, Viswanathan K, Bor Fuh C. Detection of carcinoembryonic antigen using functional magnetic and fluorescent nanoparticles in magnetic separators. $J$ Nanopart Res. 2011;13(6): 2461-2467.

29. Strekowski L, Mason CJ, Lee H, Gupta R, Sowell J, Patonay G. Synthesis of water-soluble near-infrared cyanine dyes functionalized with [(succinimido) oxy] carbonyl group. J Heterocycl Chem. 2003; 40(5):913-916.
30. Hubbuch JJ, Matthiesen DB, Hobley TJ, Thomas ORT. High gradient magnetic separation versus expanded bed adsorption: a first principle comparison. Bioseparation. 2001;10(1):99-112.

31. Ziv O, Lublin-Tennenbaum T, Margel S. Synthesis and characterization of thrombin conjugated $\gamma-\mathrm{Fe}_{2} \mathrm{O}_{3}$ magnetic nanoparticles for hemostasis. Adv Eng Mater. 2009;11(12):B251-B260.

32. Noiman T, Buzhor E, Metsuyanim S, et al. A rapid in vivo assay system for analyzing the organogenetic capacity of human kidney cells. Organogenesis. 2011;7(2):140.

33. Strekowski L, Mason CJ, Lee H, Gupta R, Sowell J, Patonay G. Synthesis of water-soluble near-infrared cyanine dyes functionalized with [(succinimido) oxy] carbonyl group. J Heterocycl Chem. 2003; 40(5):913-916.

34. Williams RJ, Lipowska M, Patonay G, Strekowski L. Comparison of covalent and noncovalent labeling with near-infrared dyes for the high-performance liquid-chromatographic determination of human serum-albumin. Anal Chem. 1993;65(5):601-605.

35. Kashin A, Tatikolov A. Spectral and fluorescent study of the interaction of anionic cyanine dyes with serum albumins. High Energy Chemistry. 2009;43(6):480-488.

36. Stopa B, Rybarska J, Drozd A, et al. Albumin binds self-assembling dyes as specific polymolecular ligands. Int J Biol Macromol. 2006;40(1): $1-8$.

37. Slowing I, Trewyn BG, Victor SYL. Effect of surface functionalization of MCM-41-type mesoporous silica nanoparticles on the endocytosis by human cancer cells. J Am Chem Soc. 2006;128(46):14792-14793.

38. Xie J, Chen K, Huang J, et al. PET/NIRF/MRI triple functional iron oxide nanoparticles. Biomaterials. 2010;31(11):3016-3022.

39. Xie J, Zhang F, Aronova M, et al. Manipulating the power of an additional phase: a flower-like $\mathrm{Au}-\mathrm{Fe}_{3} \mathrm{O}_{4}$ optical nanosensor for imaging protease expressions in vivo. ACS Nano. 2011;5(4):3043-3051.

40. Alarcón E, Aspée A, Abuin EB, Lissi EA. Evaluation of solute binding to proteins and intra-protein distances from steady state fluorescence measurements. J Photochem Photobiol B. 2012;106(0):1-17.

41. Chen T-S, Zeng S-Q, Zhou W, Luo Q-M. A quantitative theory model of a photobleaching mechanism. Chinese Physics Letters. 2003; 20(11): 1940

42. Sakuma S, Yano T, Masaoka Y, et al. In vitro/in vivo biorecognition of lectin-immobilized fluorescent nanospheres for human colorectal cancer cells. J Control Release. 2009;134(1):2-10.

43. Meller B, Rave-Fränck M, Breunig C, et al. Novel carcinoembryonicantigen-(CEA)-specific pretargeting system to assess tumor cell viability after irradiation of colorectal cancer cells. Strahlenther Onkol. 2011; 187(2):120-126. German.

44. Zou P, Xu S, Povoski SP, et al. Near-infrared fluorescence labeled anti-TAG-72 monoclonal antibodies for tumor imaging in colorectal cancer xenograft mice. Mol Pharm. 2009;6(2):428-440.

45. Lee ALZ, Dhillon SHK, Wang Y, Pervaiz S, Fan W, Yang YY. Synergistic anti-cancer effects via co-delivery of TNF-related apoptosisinducing ligand (TRAIL/Apo2L) and doxorubicin using micellar nanoparticles. Mol Biosyst. 2011;7(5):1512-1522.
International Journal of Nanomedicine

\section{Publish your work in this journal}

The International Journal of Nanomedicine is an international, peerreviewed journal focusing on the application of nanotechnology in diagnostics, therapeutics, and drug delivery systems throughou the biomedical field. This journal is indexed on PubMed Central, MedLine, CAS, SciSearch ${ }^{\circledR}$, Current Contents ${ }^{\circledR} /$ Clinical Medicine,

\section{Dovepress}

Journal Citation Reports/Science Edition, EMBase, Scopus and the Elsevier Bibliographic databases. The manuscript management system is completely online and includes a very quick and fair peer-review system, which is all easy to use. Visit http://www.dovepress.com/ testimonials.php to read real quotes from published authors. 